\title{
Heterotaxy and complex structural heart defects in a mutant mouse model of primary ciliary dyskinesia
}

\author{
Serena Y. Tan, ${ }^{1}$ Julie Rosenthal, ${ }^{1}$ Xiao-Qing Zhao, ${ }^{1}$ Richard J. Francis, ${ }^{1}$ Bishwanath Chatterjee, ${ }^{1}$ \\ Steven L. Sabol, ${ }^{1}$ Kaari L. Linask, ${ }^{1}$ Luciann Bracero, ${ }^{1}$ Patricia S. Connelly, ${ }^{2}$ Mathew P. Daniels, ${ }^{2}$ \\ Qing Yu, ${ }^{1}$ Heymut Omran, ${ }^{3}$ Linda Leatherbury, ${ }^{1,4}$ and Cecilia W. Lo' \\ ${ }^{1}$ Laboratory of Developmental Biology and ${ }^{2} \mathrm{NHLBI}$ Electron Microscopy Core Facility, National Heart Lung and Blood Institute, NIH, Bethesda, Maryland, USA. \\ ${ }^{3}$ University Children's Hospital Freiburg, Freiburg, Germany. ${ }^{4}$ Cardiology Division, Children's National Medical Center, Washington, DC, USA.
}

\begin{abstract}
Primary ciliary dyskinesia (PCD) is a genetically heterogeneous disorder associated with ciliary defects and situs inversus totalis, the complete mirror image reversal of internal organ situs (positioning). A variable incidence of heterotaxy, or irregular organ situs, also has been reported in PCD patients, but it is not known whether this is elicited by the PCD-causing genetic lesion. We studied a mouse model of PCD with a recessive mutation in Dnabc5, a dynein gene commonly mutated in PCD. Analysis of homozygous mutant embryos from 18 litters yielded $25 \%$ with normal organ situs, $35 \%$ with situs inversus totalis, and $40 \%$ with heterotaxy. Embryos with heterotaxy had complex structural heart defects that included discordant atrioventricular and ventricular outflow situs and atrial/pulmonary isomerisms. Variable combinations of a distinct set of cardiovascular anomalies were observed, including superior-inferior ventricles, great artery alignment defects, and interrupted inferior vena cava with azygos continuation. The surprisingly high incidence of heterotaxy led us to evaluate the diagnosis of PCD. PCD was confirmed by EM, which revealed missing outer dynein arms in the respiratory cilia. Ciliary dyskinesia was observed by videomicroscopy. These findings show that $D n a b c 5$ is required for the specification of left-right asymmetry and suggest that the PCD-causing Dnabc5 mutation may also be associated with heterotaxy.
\end{abstract}

\section{Introduction}

Primary ciliary dyskinesia (PCD) encompasses a wide spectrum of disorders that result from immotile or dysfunctional cilia. Cilia are highly ordered microtubule-based organelles that project from the cell surface and comprise 9 peripheral microtubule doublets decorated by outer and inner dynein arms. These dynein arms drive ciliary motion by powering sliding movement of the microtubule doublets (1). Not surprisingly, mutations causing PCD have been identified in genes encoding proteins required for ciliary biogenesis or proteins that are structural components of the cilia (2). Two of the genes commonly mutated in PCD patients are DNAH5 and DNAI1, dynein motor proteins that make up the outer dynein arms (ODAs) powering movement of the cilia (3).

PCD patients suffer from recurring respiratory infections due to inability of the ciliated respiratory epithelia to adequately propel mucus and clear foreign particles from the airway. There is also a high prevalence of male infertility due to sperm immotility. In rare instances, PCD patients exhibit hydrocephaly due to failure of the ependymal cilia to effectively move cerebrospinal fluid (4). Somewhat unexpectedly, approximately half of PCD patients have Kartagener syndrome, a condition characterized by complete mir-

Nonstandard abbreviations used: ASD, atrial septa defect; AV, atrioventricular; B6, C57BL/6J; Dnahc5, dynein, axonemal, heavy chain 5; DORV, double outlet right ventricle; EFIC, episcopic fluorescence image capture; IVC, inferior vena cava; ODA, outer dynein arm; PCD, primary ciliary dyskinesia; SEM, scanning electron microscopy/microscope; SVC, superior vena cava; TEM, transmission electron microscopy; TGA, transposition of great arteries; TOF, tetralogy of Fallot; VA, ventriculoarterial; VSD, ventricular septal defect.

Conflict of interest: The authors have declared that no conflict of interest exists. Citation for this article: J. Clin. Invest. 117:3742-3752 (2007). doi:10.1172/JCI33284. ror image reversal of organ situs known as situs inversus totalis. This association of ciliary defect with laterality reversal led Afzelius to postulate that "cilia on the embryonic epithelia have a certain position and a fixed beat direction and that their beating is somehow instrumental in determining the visceral situs" (5). The presence of such ciliated cells in the embryo and their role in situs determination has since been confirmed.

Studies using mouse embryos and other model organisms have shown that motile cilia in the early embryo play an essential role in left-right patterning (6). Insights gained from the analysis of mutations affecting ciliary structure and function have indicated a role for the cilia in left-right specification. This includes the initial breaking of symmetry and the specification of left-right organ situs along the body axes. Kif $3 A$ - and Kif3B-knockout mouse embryos have no nodal cilia, and they exhibit bilateral symmetry, indicating a failure to break symmetry (7-9). In contrast, $i v / i v$ mutants break symmetry but exhibit randomized specification of the left-right body axis, including situs solitus (normal situs) and situs inversus totalis (complete reversal of situs) (10-13). These mutants have a mutation in the left-right dynein $\operatorname{lrd}(\operatorname{Dnahc11})$, and although they have nodal cilia, the cilia are immotile.

Among PCD patients, there have been reports of heterotaxy (14), which refers to discordant organ situs in the abdominal and thoracic cavities. Heterotaxy results from indeterminate patterning of the left-right axis. In contrast to situs inversus totalis, where organ situs show complete mirror reversal or inversion from their normal left-right position, in heterotaxy the left-right positioning of the heart and great arteries, lung and liver lobes, stomach, and spleen are altered in a discordant manner. Mutations in a number of genes, such as ZIC3, LEFTYA, CRYPTIC, and ACVR2B, have been 

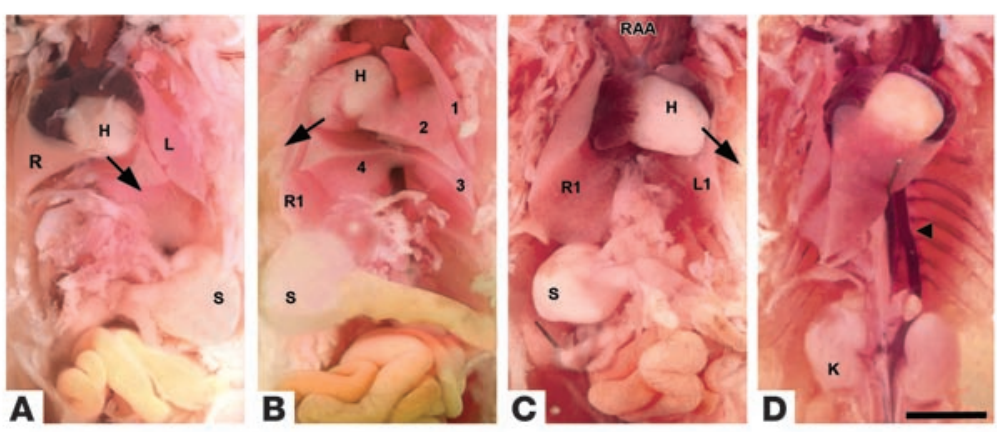

\section{Figure 1}

Situs anomalies in Dnahc5del593 mutants. (A) Situs solitus with levocardia. The right lung $(\mathrm{R})$ has 4 lobes, and the left lung $(\mathrm{L})$ has 1 . Arrow indicates direction of heart $(\mathrm{H})$ apex. The stomach (S) is on the left. (B) Situs inversus totalis with dextrocardia. Note 4 left (labeled 1, 2, 3, and 4) and 1 right (R1) lung lobes, with stomach on the right. (C and D) Heterotaxy with levocardia. Note right aortic arch (RAA), 1 lung lobe on each side (R1 and L1), and stomach on the right (C). After removal of the liver, azygos continuation (arrowhead) of the interrupted inferior vena was found (D). $\mathrm{K}$, kidney. Scale bar in D: $250 \mathrm{~mm}$ (A-D).

shown to play a role in human heterotaxy syndromes (15). In addition, a recent report showed heterotaxy is found in $6.3 \%$ of PCD patients (16). However, whether heterotaxy among PCD patients is the result of mutations causing PCD or the combined effects of other mutations or polymorphisms in the genetic background of the patient population is not known. This question is of considerable clinical importance, since heterotaxy is often associated with complex structural heart defects, which can cause significant morbidity and mortality (17). In air-breathing mammal, left-right asymmetry associated with the cardiovascular system plays a critical role in the elaboration of separate pulmonary and systemic circulation essential for the animal's survival.

In this study, we describe a new mouse model of PCD recovered in a mouse mutagenesis screen for mutations causing congenital heart disease (18). This mutant was identified with a recessive mutation in (dynein, axonemal, heavy chain 5) (Dnabc5). The human ortholog, DNAH5, is a gene often mutated in patients with PCD (3). Homozygous mutant animals exhibit situs solitus, situs inversus totalis, or heterotaxy. Analysis of preterm fetuses revealed $40 \%$ incidence of heterotaxy. Fetuses with heterotaxy exhibited varying combinations of complex structural heart defects of a defined spectrum. Given the genetic homogeneity of the mutant mouse line, these results indicate that mutations in Dnabc5 causing PCD also can cause heterotaxy. These findings suggest that PCD patients with mutations in DNAH5 should be clinically evaluated for heterotaxy and congenital heart defects.

\section{Results}

Recovery of a novel Dnahc5 mutation. As part of an ENU mutagenesis screen for recessive mutations causing congenital heart defects, we recovered a mouse mutant exhibiting situs anomalies. Homozygous animals exhibited situs inversus totalis, as well as heterotaxy (Figure 1). Mutants with heterotaxy usually also exhibited complex structural heart defects and died before or shortly after birth. To map the mutation, heterozygous mutants in the C57BL/6J (B6) background were crossed to $\mathrm{C} 3 \mathrm{H}$ mice to generate $\mathrm{B} 6 / \mathrm{C} 3 \mathrm{H}$ hybrid offspring, and these were further intercrossed to obtain embryos between E15.5 and birth. Genome scanning DNA obtained from these hybrid offspring using $\mathrm{B} 6 / \mathrm{C} 3 \mathrm{H}$ polymorphic microsatellite DNA markers mapped the mutation to chromosome 15 (18). Further fine mapping using SNPs narrowed the interval to a 7.2-Mb interval between SNP rs13482485 and rs32364740. This region contains 26 genes, including Dnabc5. As mutations in the human ortholog DNAH5 are well described in patients with situs anomalies and PCD (19), we analyzed Dnahc5 cDNA sequences and also the 79 exons of the Dnahc5 genomic DNA sequence from homozygous mutant embryos.
PCR amplification of cDNA and genomic DNA for Dnabc5 gave the expected products, except for primers targeting exons 7-17. PCR amplification of cDNA derived from these exons yielded no specific products, while amplification of genomic DNA with primers spanning exon 6 and 18 generated a $3.3-\mathrm{kb}$ amplicon from the mutant embryo DNA (Figure 2A). This compares with the expected amplicon size of 30,688 bp. Sequencing of the central 2,765 bp showed that the mutated region consisted of a large deletion of 29,755 bp spanning exons $7-17$, with the 5 ' end containing sequences from intron 6, proceeding internally to base Chr15:28,178,819 $(\mathrm{mm} 8)$, and the $3^{\prime}$ end containing sequences derived from intron 17, starting internally at base Chr15:28,208,575 (Figure 2C). Surprisingly, in this deleted interval, there was a 2,397-bp insertion composed of DNA derived from mouse chromosomes 2, 4, and X. This included both repetitive sequences and portions of exons of other genes, but these lacked known splice donor/acceptor sites (Figure 2D). In silico translation of the predicted mutant Dnabc5 mRNA showed that this novel Dnabc5 allele has an in-frame deletion of 1,779 nucleotides coding 593 amino acids from residues 267-859 at the N-terminal side of Dnabc5 - a region containing most of the heavy chain dynein interacting domain N1, but that does not include the motor domain (Figure 2C). The predicted transcript was experimentally verified by sequencing of cDNAs obtained from RT-PCR amplification of transcripts expressed in homozygous mutant embryos (Figure 2B). We refer to this mutant Dnabc5 allele as Dnahc5del593.

Situs anomalies in homozygous Dnahc5 $5^{\text {del593 }}$ mutants. With the identification of the mutation in Dnabc5, we conducted a systematic analysis of genotype versus phenotype. This survey revealed that some of the Dnabc $5^{\text {dell593 }}$ homozygous animals survived up to 2-4 weeks postnatally, exhibiting either normal situs (situs solitus; Figure $1 \mathrm{~A}$ ) or complete mirror reversal of body situs (situs inversus totalis; Figure 1B). The homozygous animals usually expired or had to be euthanized at 3-4 weeks of age, as they exhibited a domeshaped head indicative of hydrocephaly. These findings were consistent with the known role of Dnahc5 in the ependymal cilia for moving cerebral spinal fluid and maintaining patency of the aqueduct (20). A previous gene trap mouse model of PCD has also been found to have situs inversus totalis and hydrocephalus (21).

Finding both situs solitus and situs inversus totalis in the postnatal homozygous animals suggested situs specification was randomized. However, the previous examination of embryos harvested preterm also showed heterotaxy (Figure 1C). To determine the frequency of different situs presentations, 123 embryos from 18 litters were harvested at E16.5-E18.5, which consisted of 30 wild-type $\left(\right.$ Dnahc $\left.5^{+/+}\right)$, 68 heterozygous (Dnabc $5^{\text {del593/+ }}$ ), and 25 homozygous mutants (Dnahc5del593/del593). This distribution did not differ significantly 


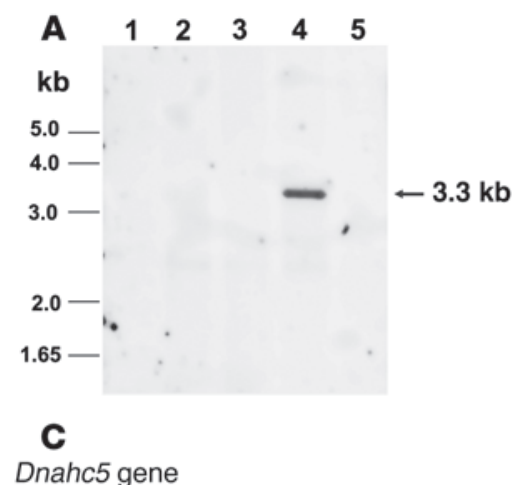

B

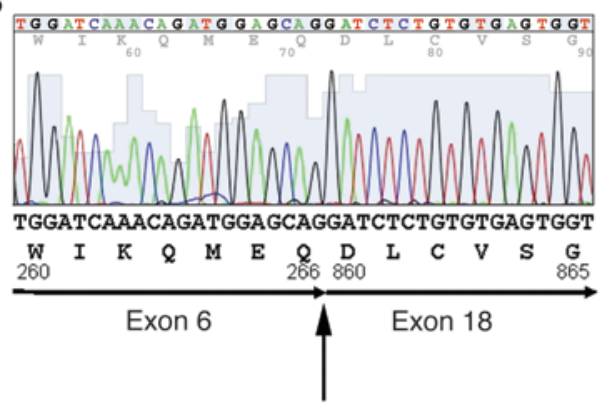

Dnahc5 gene

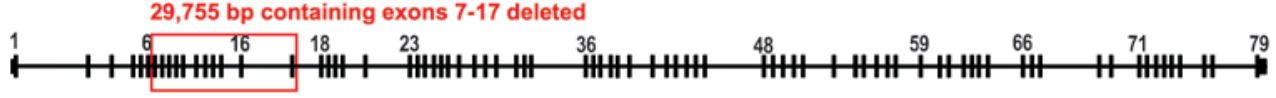

\section{Dnahc5 mRNA}

AUG $1779 \mathrm{nt}$ deleted
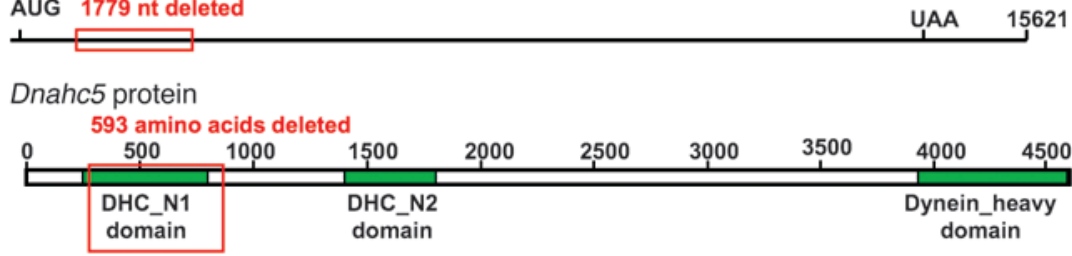

D

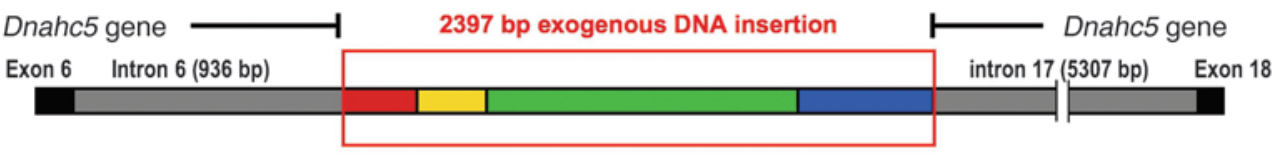

\section{Figure 2}

Dnahc5 mutation involves an in-frame DNA deletion. (A) Genomic DNA amplification using primers situated in introns 6 and 17 of the Dnahc5 gene yielded a 3.3-kb DNA fragment from a mutant embryo exhibiting heterotaxy (lane 4), while no product was obtained in DNA from normal control embryos (lanes 2, 3, and 5) or no template control (lane 1). (B) Dnahc5 cDNA sequencing showed that exon 6 is contiguous with sequence in exon 18 (arrow), indicating deletion of exons 7-17. (C) The upper bar depicts the exon/intron organization of the mouse Dnahc5 gene. Immediately below is a schematic of the Dnahc5 mRNA transcript. The red box delineates the region deleted in the Dnahc5de/593 mutant. The Pfam domains in the protein (http://pfam.sanger.ac.uk/) are shown, including dynein heavy chain N-terminal domains 1 and 2 (DHC_N1 and_N2; residues 246-804 and 1,397-1,809, respectively) and dynein heavy chain domain (residues 3,924-4,619). Two Pfam ATPase domains (residues 2,254-2,398 and 2,582-2,729) were not shown for clarity. (D) Expanded view showing region of the Dnahc5 gene containing DNA insertion derived from other chromosomes. Red: 401 bp of 31-bp tandem repeat region 99\% identical to Chr4:131,017,719131,018,166 (excluding a 47-bp gap); yellow: 265 bp of the 3 '-untranslated region of the Csnk2a1 gene (Chr2:151,972,987-151,973,251); green: 1,204 bp of the last exon of the Zbtb33 gene (ChrX:34,437,887$34,439,090$ ); blue: 516 bp of a long-terminal repeat with $100 \%$ identity at 9 locations in the mouse genome (e.g., ChrX:122,343,361-122,343,876).

from the expected Mendelian ratio. All wild-type and heterozygous mutants showed situs solitus. Interestingly, of the 25 homozygous mutant embryos, 24\% (6) exhibited situs solitus, 36\% (9) situs inversus totalis, and $40 \%$ (10) exhibited heterotaxy. We define heterotaxy as any deviation from situs solitus or situs inversus totalis, whether the changes were observed in the cardiac, pulmonary, or abdominal anatomy. These observations show this Dnahc5 mutation generates complete reversal of handedness as in situs inversus totalis at the same rate as the incidence of heterotaxy.

In situs solitus, the heart apex points leftward (levocardia) and the aortic arch is leftward (Figure 1A). The aorta is posterior and right of the pulmonary artery at the base of the heart. Both superior vena cavae (SVCs) return to the right atrium - the left SVC via the coronary sinus and the inferior vena cava (IVC) returns to the right atrium. There are 4 left and 1 right lung lobes, and the stomach, pancreas, and spleen are on the left. Only the left liver lobe is visible posterior to the medial lobe, which straddles both sides of the body. In situs inversus totalis, there is complete mirror-image reversal of organ situs in the thoracic and abdominal cavities. For example, the heart apex is pointed to the right (dextrocardia), with a right-sided aortic arch, and the stomach, spleen, and pancreas are on the right (Figure 1B). In embryos with heterotaxy, we observed a wide range of situs anomalies (Table 1). Some had 1 lung lobe on each side of the chest cavity, indicative of left pulmonary isomerism. There were also examples of symmetrical bilobed liver, midline stomach and pancreas, as well as azygos continuation of IVC (Figure 1, C and D, and Table 1). Abnormal heart situs and complex structural heart defects were also observed in many of the mutants exhibiting heterotaxy (Table 1). Light blue in Table 1 indicates normal situs; pink, inverted situs; gray, right-left isomerism; and green, midline position or otherwise anomalous position/connection. It is interesting to note that isomerism (gray) and the more anomalous pathologies (green) were more frequently associated with levocardia.

Complex structural heart defects in heterotaxic embryos. To evaluate the structural heart defects associated with heterotaxy, the hearts were further analyzed using serial section histology carried out by episcopic fluorescence image capture (EFIC) followed by $3 \mathrm{D}$ reconstruction. To broaden the analysis for structural heart defects, an additional 8 homozygous mutant embryos exhibiting heterotaxy were included in this histological analysis. Of the 18 hearts examined, 6 showed levocardia, 10 had dextrocardia, and 2 exhibited midline position or mesocardia (Table 2). To delineate the complex structural heart defects, we adapted Van Praagh's segmental analysis (22), in which the heart is subdivided into atrial, ventricular, and arterial segments, with each segment containing 2 components - the right versus left atrial chambers, the right versus left ventricular chambers, and the aortic versus pulmonary trunks (Table 1 ). In addition, we examined 


\section{Table 1}

Cardiovascular, pulmonary, and abdominal phenotypes associated with heterotaxy

\begin{tabular}{|c|c|c|c|c|c|c|c|c|c|c|c|c|}
\hline No. & Apex ${ }^{A}$ & $\begin{array}{l}\text { Atrial } \\
\text { situs }^{B}\end{array}$ & Loopc & $\begin{array}{c}\text { AV } \\
\text { Connection }\end{array}$ & $\begin{array}{c}\text { VA } \\
\text { AlignmentE }\end{array}$ & $\begin{array}{c}\text { Ao-PA } \\
\text { relation }\end{array}$ & $\begin{array}{l}\text { Segment } \\
\text { analysis }\end{array}$ & $\begin{array}{c}\text { Septal } \\
\text { defects }^{G}\end{array}$ & $\begin{array}{l}\text { Aortic } \\
\operatorname{arch}^{\mathrm{H}}\end{array}$ & $\begin{array}{l}\text { Venous } \\
\text { return' }\end{array}$ & $\begin{array}{l}\text { Lung } \\
\text { situs }^{J}\end{array}$ & Abdomen ${ }^{k}$ \\
\hline 1 & Lev & SS & D & AVC & TOF & $\mathrm{A} 0>\mathrm{PA}$ & $\{S, D, S\}$ & $\begin{array}{c}\text { pASD, VSD } \\
(0 A 0)\end{array}$ & LAA & Normal & 1L:1R, LPI & $\mathrm{L}(\mathrm{St} / \mathrm{Sp} / \mathrm{P})$ \\
\hline 2 & Lev & LAI & $D(s-i)$ & AVC & DORV & Ao ant & $\{A, D, D\}$ & ASD, VSD & IAA (B) & $2 \mathrm{AZYs}$ & ND & ND \\
\hline 3 & Lev & LAI, CA & D & UAVC (LV) & DORV & $\begin{array}{l}\text { Parallel } \\
\text { Ao dex }\end{array}$ & $\{A, D, D\}$ & VSD(Ao) & LAA & AZY, 2 HVs & 1L:1R, LPI & $\begin{array}{l}\mathrm{R}(\mathrm{St} / \mathrm{Sp} / \mathrm{P}) \\
\text { SymLiv }\end{array}$ \\
\hline 4 & Lev & LAI, CA & D & AVC & D-TGA & Ao ant & $\{A, D, D\}$ & 2 VSDs & LAA, aRSC & Normal & 1L:1R, LPI & $\begin{array}{l}\text { MidSt, } \\
\text { R(SpP) }\end{array}$ \\
\hline 5 & Lev & LAI, CA & $L(s-i)$ & 1 AVV (MV) & CC & Ao lev & $\{A, L, I\}$ & VSD & RAA & AZY, 2 HVs & 1L:1R, LPI & $\mathrm{R}(\mathrm{St} / \mathrm{Sp} / \mathrm{P})$ \\
\hline 6 & Lev & RAl & $D(s-i)$ & 1 AVV (MV) & DORV & $\begin{array}{l}\text { Parallel } \\
\text { Ao lev }\end{array}$ & $\{A, D, L\}$ & $\begin{array}{l}P+S A S D \\
\text { VSD }(A 0)\end{array}$ & LAA & 2 IVCs & 1L:1R, ND & $\begin{array}{l}\mathrm{R}(\mathrm{St} / \mathrm{Sp} / \mathrm{P}) \\
\text { SymLiv }\end{array}$ \\
\hline 7 & Dex & SS & D & UAVC (RV) & L-TGA & Ao ant lev & $\{S, D, L\}$ & ASD, VSD & RAA & Normal & 4L:1R, SI & $\mathrm{L}(\mathrm{Sp} / \mathrm{P} / \mathrm{St})$ \\
\hline 8 & Dex & SI & $L(s-i)$ & UAVC (LV) & DORV & Ao ant & $\{I, L, L\}$ & pASD & RAA & AZY, 2 HVs & 1L:1R, LPI & $\begin{array}{c}\text { L(St/Sp/P) } \\
\text { SymLiv }\end{array}$ \\
\hline 9 & Dex & $\mathrm{SI}$ & $\mathrm{L}$ & 2 AVVs & TOF & Inv & $\{I, L, \mid\}$ & VSD (OAo) & RAA & $A Z Y$ & 4L:1R, SI & RSt/MalP/aSp \\
\hline 10 & Dex & SI & L & 2 AVVs & $\mathrm{CC}$ & Inv & $\{I, L, I\}$ & None & RAA & AZY, 2 HVs & 4L:1R, SI & RSt, No(Sp/P) \\
\hline 11 & Dex & $\mathrm{SI}$ & L & 2 AVVs & DORV & Ao ant & $\{l, L, L\}$ & VSD (MPA) & RAA, HTA & Inv & 4L:1R, SI & $\mathrm{R}(\mathrm{StSpP})$ \\
\hline 12 & Dex & SI & L & 2 AVVs & $\mathrm{CC}$ & Ao ant & $\{l, L, L\}$ & None & RAA & $A Z Y$ & 3L:3R:1M, RPI & $\mathrm{R}(\mathrm{St} / \mathrm{Sp} / \mathrm{P})$ \\
\hline 13 & Dex & SI & L & 2 AVVs & CC & Inv & $\{I, L, \mid\}$ & 2 VSDs & RAA & IVC, 2 HVs & $1 \mathrm{~L}: 1 \mathrm{R}, \mathrm{SI}$ & $\begin{array}{l}\text { RSt/MidP, } \\
\text { aSp/SymLiv }\end{array}$ \\
\hline 14 & Dex & $\mathrm{SI}$ & $\mathrm{L}$ & 2 AVVs & CC & Inv & $\{I, L, I\}$ & None & RAA & Inv & 4L:1R, SI & $\mathrm{R}(\mathrm{StSpP})$ \\
\hline 15 & Dex & SI & L & UAVC (LV) & D-TGA & Ao ant & $\{l, L, D\}$ & ASD, VSD & LAA & Inv & $4 \mathrm{~L}: 1 \mathrm{R}, \mathrm{SI}$ & $\mathrm{R}(\mathrm{St} / \mathrm{Sp} / \mathrm{P})$ \\
\hline 16 & Dex & SI & L & 2 AVVs & CC & Inv & $\{I, L, \mid\}$ & None & RAA & $A Z Y$ & 4L:1R, SI & $\mathrm{R}(\mathrm{St} / \mathrm{Sp} / \mathrm{P})$ \\
\hline 17 & Mes & SS & D & 2 AVVs & D-TGA & $\begin{array}{l}\text { A0 }>\text { PA } \\
\text { Ao ant }\end{array}$ & $\{S, D, D\}$ & iVSD & LAA & Normal & $1 \mathrm{~L}: 2 \mathrm{R}, \mathrm{SS}$ & $\mathrm{L}(\mathrm{St} / \mathrm{Sp} / \mathrm{P})$ \\
\hline 18 & Mes & SS & D & 2 AVVs & DORV & Normal & $\{S, D, S\}$ & oVSD, pASD & LAA & Normal & 1L:4R, SS & ND \\
\hline
\end{tabular}

Light blue, normal situs; pink, inverted situs; gray, left-right isomerism; green, anomalous; ND, not determined. ADex, dextrocardia; Lev, levocardia; Mes, mesocardia. BSS, situs solitus; SI, situs inversus totalis; RAI, right atrial isomerism; LAI, left atrial isomerism; CA, common atrium. CD, D-loop; L, L-loop; $\mathrm{S}$-i, superior-inferior positioning of ventricles. ${ }^{\mathrm{A} A V V}$, atrioventricular valves; AVC, common atrioventricular canal; MV, mitral valve morphology; UAVC, unbalanced AVC, committed to LV or RV. ECC, concordant; D-/L-TGA, D-/L-transposition of great arteries. FInv, inverted; Ao, aorta; ant, anterior; dex, dextro, i.e., Ao displaced toward the right and anterior of the PA; lev, levo, i.e., Ao displaced on the left and anterior of the PA; Ao>PA, reduced gauge of PA compared with Ao, which is associated with Tetralogy of Fallot. Gi/oVSD(Ao/MPA), inlet/outlet ventricular septal defect committed to aorta/main pulmonary artery; OAo, overriding aorta; ASD, atrial septal defect, primum (p) or secundum (s). HRAA, right-sided aortic arch; LAA, left-sided aortic arch; HTA, hypoplastic transverse arch; aRSC, aberrant right subclavian artery; IAA (B), interrupted aortic arch type B. 'AZY, azygos continuation of interrupted inferior vena cava; HV, hepatic vein. JL:R:M, number of left:right:middle lung lobes; bronchial tree situs indicated by L/RPI (left/right pulmonary isomerism). KSt, stomach; Sp, spleen; P, pancreas — either left (L) or right sided (R); aSP, asplenia; Mal, malpositioned; Mid, midline; Hypo, hypoplastic; SymLiv, symmetric liver.

the atrioventricular (AV) and ventriculoarterial (VA) connections, venous drainage, aortic arch connections, and septal defects.

Atrial situs. As mouse atria do not exhibit hallmark features seen in human left versus right atrial appendages, atrial situs was classified according to the pattern of venous return and the presence of a coronary sinus. If the ipsilateral SVC of an atrium returned directly to it, it was designated a morphologic right atrium. If its ipsilateral SVC returned via a coronary sinus to the contralateral atrium, it was designated a morphologic left atrium. In left atrial isomerism, there is often a common atrium to which both SVCs return. In right atrial isomerism, each SVC returns directly to its ipsilateral atrium, and in addition, each atrium has an inferior venous connection as well. Designating the atrial situs as such, we were able to classify all atrial pairs, yielding 4 situs solitus, 9 situs inversus, 4 with left atrial isomerism (Figure 3C) and 1 with right atrial isomerism (Figure 4). All cases of atrial isomerism occurred in hearts with levocardia, and both hearts with mesocardia had situs solitus atrial arrangement (Table 1).

Ventricular loop. The ventricular morphology was determined by a combination of hallmarks: the morphologic left ventricle is charac- terized by the attachment of papillary muscles to the free wall. The roof of the right ventricle typically has a band of muscle known as the trabecula septomarginalis. The lack of conal tissue between the ventricular inflow and outflow valve is typical of a mitral and aortic valve, respectively. In contrast, conal tissue is typically seen between the tricuspid and pulmonic valve. Using these criteria, 14 of the hearts had ventricles that could be classified (Table 1). The loop direction was concordant with the orientation of the apex in all but 1 of these hearts. However, the latter heart exhibited AV concordance, as indicated by dextrocardia presenting with atrial situs solitus and ventricular D-loop. The remaining 4 hearts had a superior-inferior, rather than left-right, arrangement of ventricles (Figure 5, A-D, and Figure 4B). In all 4 hearts, the morphologic right ventricle was positioned superiorly and was the larger of the 2 ventricular chambers (Figure 5, C and D). In 2 cases, the left inferior ventricle was displaced leftward and hence designated " $D$ superior-inferior," and in the other 2 , the left ventricle was rightward, hence "L superior-inferior" (Table 1). Independent of leftright or superior-inferior positioning of ventricles, the 18 hearts were almost equally divided between D- and L-loops (Table 2). 


\section{Table 2}

Summary of heart, lung, stomach, situs associated with heterotaxy

\begin{tabular}{|c|c|c|c|c|c|c|c|c|c|c|c|}
\hline $\begin{array}{l}\text { Heart } \\
\text { apex }\end{array}$ & $\begin{array}{l}\text { Atrial } \\
\text { situs }\end{array}$ & Loop & $\begin{array}{c}\text { AV } \\
\text { connection }\end{array}$ & $\begin{array}{c}\text { AV } \\
\text { alignment }\end{array}$ & $\begin{array}{c}\text { Ao-PA } \\
\text { position }^{A}\end{array}$ & $\begin{array}{l}\text { Septal } \\
\text { defect }\end{array}$ & $\begin{array}{l}\text { Aortic } \\
\operatorname{arch}^{B}\end{array}$ & $\begin{array}{l}\text { Venous } \\
\text { return }\end{array}$ & $\begin{array}{c}\text { Bronchial } \\
\text { tree }^{\mathrm{C}}\end{array}$ & $\begin{array}{l}\text { Lung } \\
\text { lobes }^{D}\end{array}$ & Abdomen \\
\hline $\operatorname{Dex}(10)$ & SS (4) & $\mathrm{D}(6)$ & 2 AVVs (9) & $\mathrm{CC}(6)$ & Normal (2) & None (4) & CC (15) & Normal (8) & SS (2) & CC (14) & CC (6) \\
\hline Lev (6) & SI (9) & L (8) & $1 \mathrm{AVV}(2)$ & TGA (4) & $\operatorname{lnv}(6)$ & ASD (2) & $\mathrm{DC}(3)$ & AZY (4) & SI (8) & $\mathrm{DC}(2)$ & DC (5) \\
\hline \multirow[t]{2}{*}{ Mes (2) } & LAI (4) & $D(s-i)(2)$ & UAVC (4) & DORV (6) & D-Mal (5) & VSD (7) & & AZY/HV (4) & LPI (5) & ND (2) & Mal (4) \\
\hline & RAl (1) & L (s-i) (2) & $\operatorname{AVC}(3)$ & TOF (2) & L-Mal (5) & ASD/VSD (5) & & Other (2) & RPI (1) & & ND (3) \\
\hline
\end{tabular}

Numbers in parentheses indicate number of embryos exhibiting phenotype. ${ }^{A} \mathrm{D} / \mathrm{L}-\mathrm{Mal}$, D-/L-malpositioned. ${ }^{\mathrm{B} C C} / \mathrm{DC}$, concordant/discordant vs. heart loop. $\mathrm{C}$ In 2 embryos, bronchial tree patterning was not evaluated. ${ }^{\mathrm{C} C C / D C}$, concordant or discordant with bronchial situs. ${ }^{\mathrm{E} C C / D C}$, concordant/discordant with cardiac situs; Mal, malpositioning or malformation of 1 or more of the abdominal organs.

Aorta-pulmonary artery positioning. The position of the aorta is right and posterior in relation to the pulmonary artery in normal hearts, or left and posterior in hearts with mirror-image reversal of situs. D-malposition occurs when the aorta is displaced toward the right and anterior relative to the pulmonary artery, and L-malposition when the aorta is displaced toward the left and anterior in a heart with inverted situs. Ten of the 18 hearts had malpositioned aortas, which were equally divided between D-malpositioning (Figure 6, A and C) and L-malpositioning (Figure 6B; Tables 1 and 2).

$A V$ connection. Variations from the normal AV connection comprising $2 \mathrm{AV}$ valves included a single $\mathrm{AV}$ valve and a common $\mathrm{AV}$ canal that was either balanced or unbalanced, the latter being committed either to the morphologic left or right ventricles. The 18 hearts were equally divided between those that had normal AV connections and those that did not (Table 2). Hearts with atrial or ventricular segments that deviated from normal situs or a simple situs inversion, such as with atrial isomerism or superior-inferior positioning of ventricles, invariably had AV canal defects (Figure $3 \mathrm{D}$ and Figure 5, E and F). Two of the 4 hearts with superior-inferior ventricles had a single $\mathrm{AV}$ valve of mitral valve morphology connecting a common atrium (left atrial isomerism) or 2 atria with primum and secundum atrial septal defects (ASDs) (right atrial isomerism) to the inferiorly positioned morphologic left ventricle. In hearts with normal or simply inverted atria and ventricles, AV canal defects were accompanied by VA malformations, e.g., transposition of great arteries (TGA).

\section{Figure 3}

Histological analysis of cardiac anomalies. (A and B) Outflow tract defects. (A) Posterior view of heart exhibiting dextrocardia with TOF. The aorta (Ao) overrides the VSD and is continuous with both the leftsided morphologic right ventricle (mRV) and the right-sided morphologic left ventricle $(\mathrm{mLV})$. The pulmonary artery $(\mathrm{PA})$ is narrower than the Ao. The mitral valve (MV) is continuous with the aortic valve and opens into the mLV. (B) Posterior view of heart with levocardia and DORV. Both the PA and Ao open into the right-sided mRV. (C and D) Atrial isomerism and common AV canal. (C) Frontal view showing left atrial isomerism with both SVCs entering the common atrium (CA) laterally. Rather than the right SVC entering the roof of the right atrium and the left SVC entering the right atrium via the coronary sinus, the SVCs return symmetrically to the sides of the CA, which serves as both the right atrium and the coronary sinus. Two hepatic veins (HVs) also return directly via midline to the common atrium inferiorly. (D) Resectioned 2D transverse slice from 3D reconstruction, showing a common AV canal (AVC) with a single AV leaflet. Note the symmetry of the 2 SVCs. Scale bars: $200 \mu \mathrm{m}$.
VA alignment. VA alignment is said to be concordant if the morphologic left ventricle connects to the aorta and the morphologic right ventricle to the pulmonary artery. Ten of the 18 hearts had discordant VA alignment, of which double outlet right ventricle (DORV) was the most common (Figure 3B, Figure 6D, and Table 2). Three of the 4 hearts with superior-inferior positioning of ventricles had DORV accompanied by ventricular septal defects (VSDs) (Figure 5C). All 6 D-looped hearts had abnormalities in VA alignment: 2 were DORV (Figure 3B), 2 were D-TGA (Figure 6, A and C, and Figure 7), 1 was L-TGA (Figure 6B), and 1 was tetralogy of Fallot (TOF) (Figure $3 \mathrm{~A}$ and Table 1). Of the $8 \mathrm{~L}$-looped hearts, 6 had concordant VA alignment. Of these, 1 had an L-malposition of the great arteries, and 1 had TOF. Only 2 of the L-looped hearts showed discordance in VA alignment, 1 having D-TGA and the other DORV (Figure 6D).

Aortic arch. The direction of the aortic arch was concordant with the ventricular loop in 15 of the 18 hearts (Table 2), in that a D-looped ventricle had a left aortic arch or an L-looped ventricle had a right aortic arch. There was 1 example each of a D-looped ventricle discordant with a right aortic arch and an L-looped ventricle discordant with a left aortic arch (Table 1, nos. 7, 15). There was 1 case of a type B interrupted aortic arch (Table 1, no. 2). Other aortic anomalies were infrequent, with 1 case of hypoplastic transverse arch (Table 1 , no. 11) and 1 aberrant right subclavian artery off the descending aorta (Table 1, no. 4).

Venous drainage. By far the most common venous anomaly was azygos continuation of interrupted IVC (Figure 1D), seen in 8

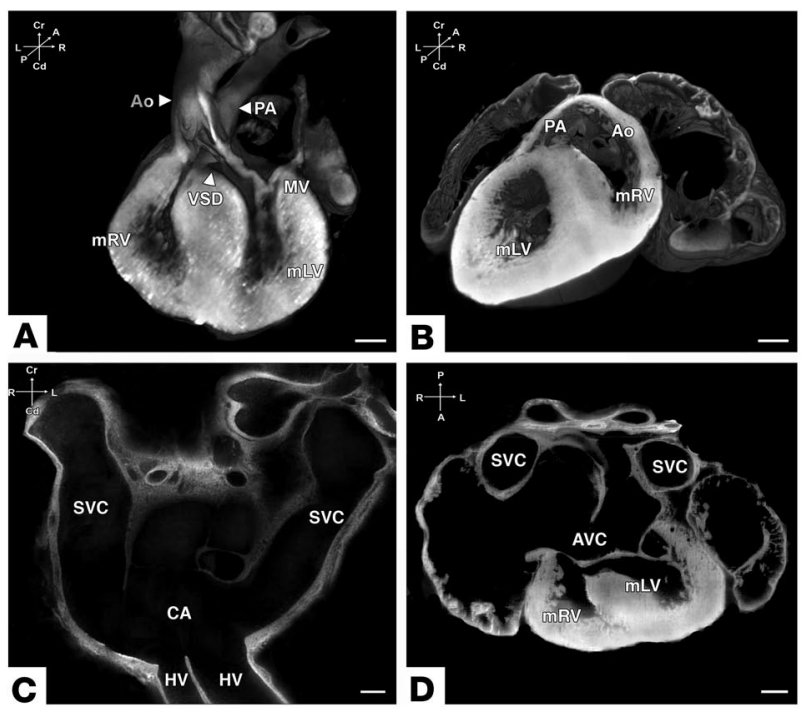




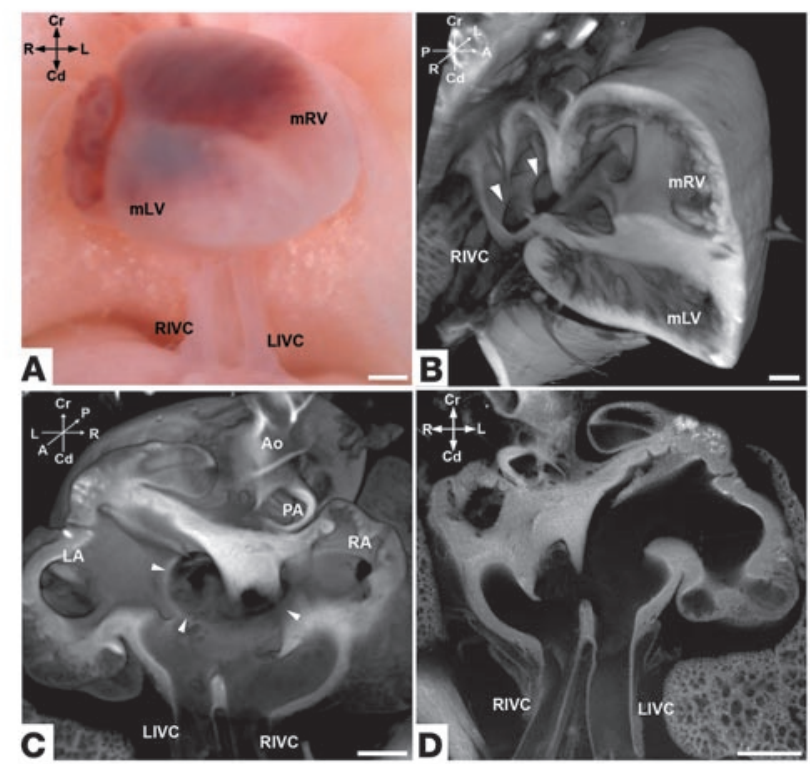

mutants (Table 2). Duplicated IVC was seen twice, giving a total of 10 hearts with venous anomalies. Eight of the 10 were associated with either pulmonary or abdominal situs abnormality, e.g., left pulmonary isomerism, malpositioned pancreas, or symmetric liver (Table 1). Only in 1 case was the venous anomaly found as an isolated defect (Table 1, no. 16).

Cardiac-pulmonary and cardiac-abdominal situs. Analysis of the bronchial tree patterning showed substantial correlation with atrial patterning (Table 1). Nine of the 10 heterotaxic embryos with either situs solitus or situs inversus bronchial tree patterning had the corresponding atrial arrangement. All of the embryos that had left atrial isomerism also had left isomerism of the bronchial tree. However, the converse was not true, as 3 of the embryos with left isomerism of the bronchial tree exhibited atrial arrangement that was either situs solitus or situs inversus. In 4 of the embryos, we observed discordance between the direction of heart loop and the situs of stomach, spleen, and pancreas. Thus, a D-looped heart was associated with a right stomach, spleen, and pancreas, and the

\section{Figure 5}

Superior-inferior ventricles and AV canal defect. (A) Superior-inferior ventricles with congested superior ventricle and atrial appendages with $\mathrm{Ao}$ and PA side by side. Note this same heart is shown in different views in Figure 4. (B) 3D model shows $\mathrm{mLV}$ is inferior and rightsided, while the $\mathrm{mRV}$ is superior and left-sided. (C) 3D reconstruction in an apical orientation looking anteriorly at 2 papillary muscles in the smaller, inferior $\mathrm{mLV}$, as indicated by the 2 arrowheads. There is also a small, muscular VSD present near the apex. (D) Anterior view shows a larger, superior $\mathrm{mRV}$ with the septomarginalis $(\mathrm{M})$ between the superior ventricular outflow tract and the body of the right ventricle. The aortic and pulmonic valves (AV and PV) both arise from the $\mathrm{mRV}$, giving rise to a DORV with a VSD. The arrowhead indicates the AV canal defect. (E) Anterior view of 3D EFIC reconstruction illustrates side-by-side, semilunar valves of similar height associated with DORV. The bicuspid aortic valve (AV) is on the left, and the tricuspid pulmonic valve (PV) is on the right. An AV canal defect (AVC) is situated at the crux of the heart. (F) Frontal 2D section showing the same AVC as in E entering both the $\mathrm{mRV}$ and the $\mathrm{mLV}$, with a VSD denoted by the arrow. LA, left atrium. Scale bars: $500 \mu \mathrm{m}$.

\section{Figure 4}

Images of anomalous venous return. (A) Two inferior venous structures are seen in this mutant. (B) 3D reconstruction show opening of dual cava (arrowheads) into the base of the atria, with the right IVC (RIVC) most anterior. The mRV is positioned superiorly and the $\mathrm{mLV}$ inferiorly. (C) 3D reconstruction looking posteriorly at the left and right IVCs connecting to the base of the 2 morphologic right atria suggests right atrial isomerism. The arrowheads highlight the border of the AV canal, from which blood empties into both the $\mathrm{mLV}$ and $\mathrm{mRV}$. Side-byside outflows are also present with the aorta anterior and on the left and the PA on the right. (D) Original 2D image looking anteriorly at the 2 atria. Note the presence of duplicated IVC entering into the base of both morphologic right atria. RA, right atrium. Scale bars: $500 \mu \mathrm{m}$.

converse was found for an L-looped heart. These embryos were invariably associated with pulmonary isomerism (Tables 1 and 2).

Analysis of ciliary ultrastructure, orientation, and function. The finding of $40 \%$ heterotaxy associated with this Dnabc $5^{d e l 593}$ mutation is striking and somewhat unexpected. This compares with only a $6.3 \%$ incidence of heterotaxy in a recent clinical study of 337 PCD patients (16). To ascertain whether the Dnabc5 $5^{\text {del } 593}$ mutation is indeed a bona fide model of PCD, we analyzed ciliary structure and function in the respiratory airway. For this analysis, trachea were isolated from homozygous animals $2-4$ weeks old and processed for scanning sand transmission electron microscopy (SEM and TEM).

SEM showed an orderly arrangement of the cilia in wild-type and heterozygous animals such that all of the cilia were oriented in a similar direction, presumably aligned with the direction of mucus flow in the airway (Figure 8, A and B). In contrast, among homozygous mutants, a wide variation was observed, ranging from marked disorganization of cilia with no obvious alignment (Figure 8C) to organized cilia similar to those seen in wild-type animals (compare with Figure 8A; and data not shown). SEM

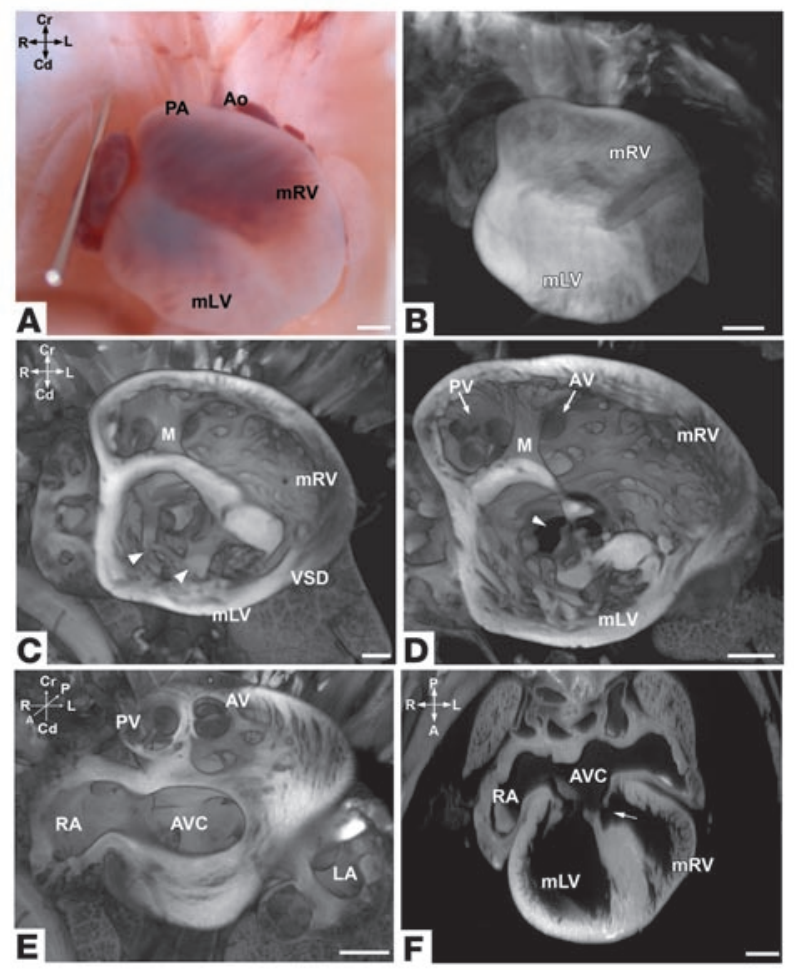



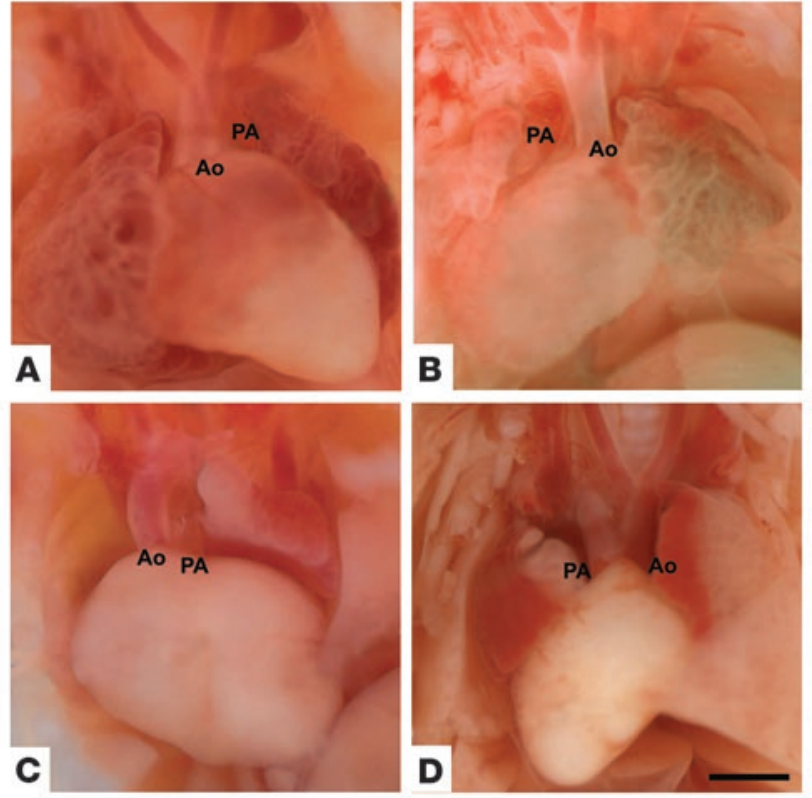

analysis of embryos at E7.75-E8.0 revealed that cells at the embryonic node are monociliated in both wild-type and homozygous mutant embryos (Figure 8, J and $\mathrm{K}$ )

TEM analysis of the wild-type tracheal cilia showed the expected 9 plus 2 arrangement of microtubules (Figure 8, D-I), with outer and inner dynein arms decorating the peripheral doublets (Figure 8G). PCD patients with mutations in DNAH5 typically have cilia with missing ODAs (3). In our mutants, the number of ODAs was markedly reduced (Figure 8I), with $7.5 \pm 2.1$ ODAs per cilium seen in wild-type animals and $0.29 \pm 0.56$ and $5.7 \pm 2.5$ ODAs per cilium in homozygous and heterozygous mutants, respectively. A frequency distribution plot of the number of ODAs per cilium showed a significant difference between all 3 genotypes (Figure 9), indicating that the Dnabc5 $5^{\text {del } 593}$ allele causes ODA deficiency, as DNAH5 mutations do in PCD patients. We also examined the variability in ciliary orientation by drawing a line through the central doublet in a cross-section view of the cilia and calculating the angular variance relative to the reference line (Table 3; see Methods), a measurement often used in the clinical diagnosis of PCD (23). As expected, the cilia of homozygous mutants showed significantly greater variation in orientation than wild-type cilia, in agreement with the qualitative SEM analysis (Table 3).

To examine ciliary function in the airway epithelia, we used another standard assay for PCD involving motion analysis of the tracheal epithelia by videomicroscopy. Wild-type tracheal epithelia exhibited synchronous movement of the cilia, while the airway

\section{Figure 7}

EFIC imaging of heart exhibiting mesocardia with inlet VSD and D-TGA. (A) EFIC 3D reconstruction showing anterior view of same heart shown in Figure 3C. (B) D-TGA is observed with the Ao on the right and connected with the mRV. The PA is on the left and connected with the mLV. (C-F) EFIC frontal section images of the same heart. The PA and Ao, distally septated (C), are seen to fuse at their attachment to the heart (D). The resultant inlet VSD (IVSD) is straddled by a single $A V$ valve leaflet (AVL) (E). The PA continues into the $L V$, and the Ao into the RV, resulting in D-TGA (F). Scale bars: $500 \mu \mathrm{m}$.

\section{Figure 6}

Necropsies showing outflow tract defects in Dnahc5del593 mutants. (A) D-malposition of great vessels is shown with levocardia with anterior and rightward Ao relative to the PA. EFIC analysis showed that this heart was D-looped with D-TGA. (B) Dextrocardia with anterior and leftward aorta indicating L-malposition of great vessels. EFIC showed this was a D-looped heart with L-TGA. (C) Mesocardia with parallel great vessels and right-sided aorta, i.e., D-malposition. EFIC showed that this heart was D-looped with D-TGA. (D) Dextrocardia with parallel great vessels and left-sided aorta, i.e., L-malposition. EFIC showed that this heart was D-looped with DORV. Scale bar: $1 \mathrm{~mm}$.

epithelia of homozygous mutants were immotile, or slow and dyskinetic. We quantitatively assessed the effectiveness of ciliary beating using fluorescent beads deposited above the epithelia. Motion analysis showed a marked reduction in both the velocity and directionality of bead displacement by the homozygous mutant tracheal epithelia, indicating a marked reduction in net fluid flow (Table 3). However, the airway epithelia of wild-type and heterozygous animals showed no significant difference in bead displacement, consistent with the absence of any situs defects in such animals (Table $3)$. Together these findings confirm that the $D n a b c 5^{\text {dels93 }}$ allele is a recessive mutation that models $\mathrm{PCD}$.

\section{Discussion}

We recovered a mouse line with a recessive mutation in Dnabc5, the mouse ortholog of $D N A H 5$, a gene frequently mutated in patients with PCD (3). Homozygous mutants exhibited situs inversus totalis, ciliary dyskinesia in the respiratory airway, and hydrocephalus - defects consistent with PCD. In addition, heterotaxy with complex heart defects was frequently observed. Although our mutant was recovered as part of an ENU mutagenesis screen, the mutation was atypical, consisting of a large deletion of nearly $30 \mathrm{~kb}$
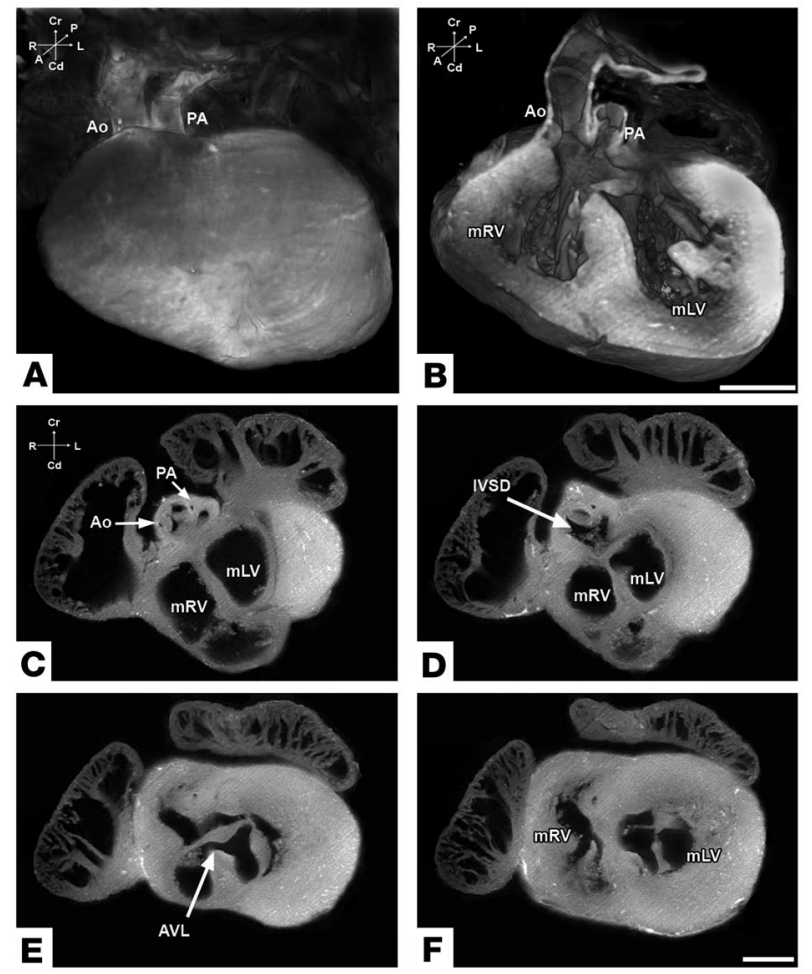

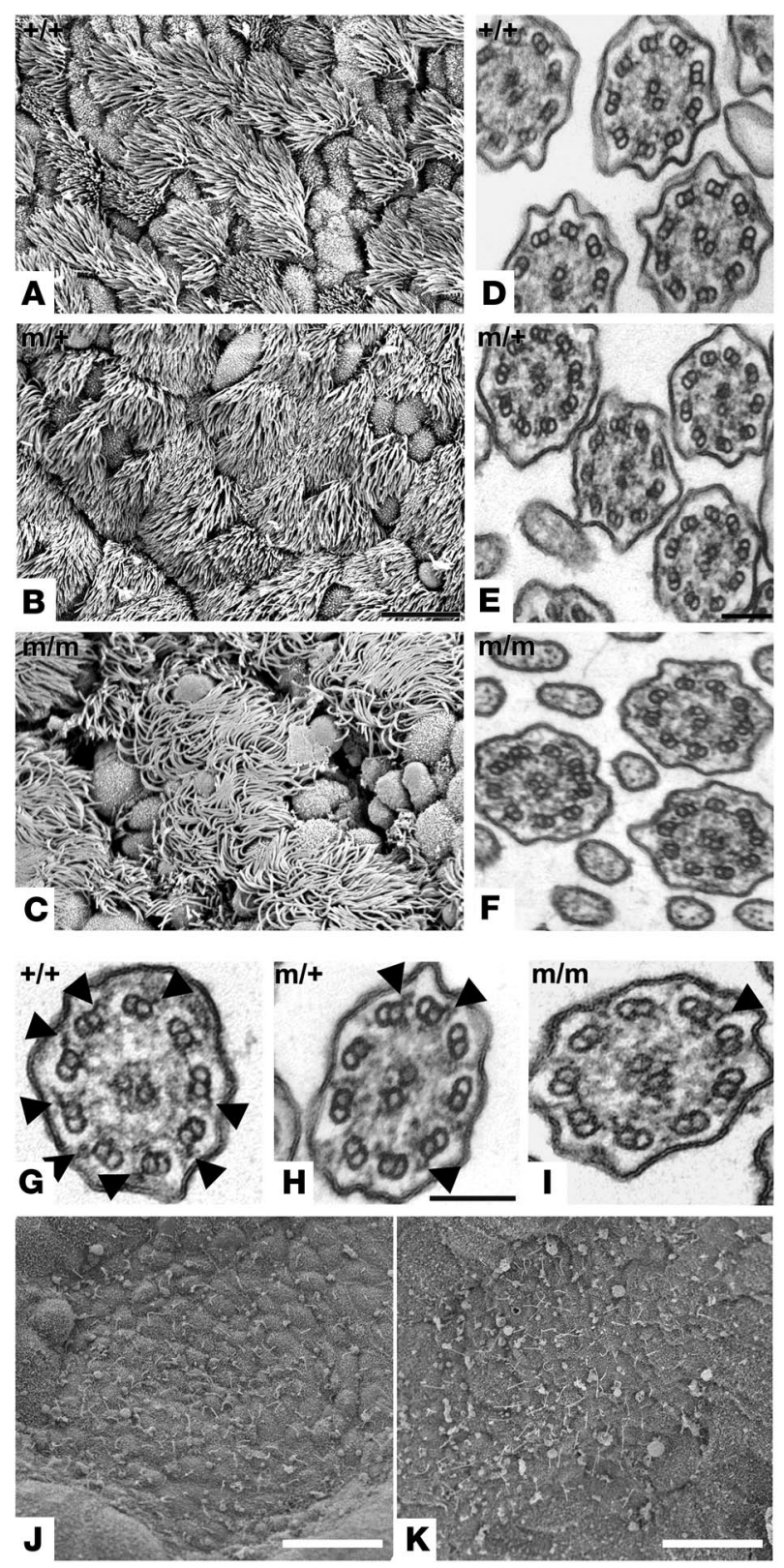

of genomic sequences spanning exons 7 through 17 and the concomitant insertion of $2.4 \mathrm{~kb}$ of genomic sequences from other chromosomes. This resulted in the deletion of 593 amino acids encompassing the heavy chain dynein interacting domain N1, leaving intact the dynein motor domain. TEM analysis showed missing ODAs in homozygote mutants, similar to the findings in patients with DNAH5 mutations (3). Although heterozygote animals exhibited a modest but significant reduction in ODAs, there was no change in net ciliary flow. Consistent with this, situs anomalies were never seen in any of the heterozygote adult or newborn animals. Together, these findings indicate this novel Dnabc5 mutation is a bona fide model of human PCD.

Another mutant mouse line with an insertional mutation in Dnabc5 has been reported previously: $M d n a b 5^{-/-}$(21). It consists of a transgene insertion associated with the deletion of exons 16-22 of Dnabc5, resulting in a fusion transcript with a stop codon down-

\section{Figure 8}

SEMs and TEMs of tracheal epithelial cilia. (A-C) SEMs of tracheal epithelial cilia from wild-type $(+/+)(\mathbf{A})$, heterozygote $(\mathrm{m} /+)(B)$, and homozygote mutant $(\mathrm{m} / \mathrm{m})(\mathbf{C})$ animals. Note the marked disorientation in the $\mathrm{m} / \mathrm{m}$ cilia. Scale bar in B: $500 \mu \mathrm{m}$ (A-C). (D-F) TEMs show +/+ cilia (D) with ODA on almost every doublet; $\mathrm{m} / \mathrm{m}$ cilia (F) with 1 or no ODA; and $\mathrm{m} /+$ cilia (E), some well-populated with ODAs and others with reduced numbers of ODAs. Scale bar in E: $500 \mathrm{~nm}$ (D-F). (G-I) High-powered TEMs of $+/+(\mathbf{G}), \mathrm{m} /+\mathbf{( H})$, and $\mathrm{m} / \mathrm{m}$ (I) tracheal epithelial cilia, with black arrowheads indicating ODAs. Scale bar in $\mathbf{H}: 500 \mathrm{~nm}(\mathbf{G}-\mathbf{I})$. ( $\mathbf{J}$ and K) SEMs of E7.75 wild-type (J) and homozygous (K) mutant embryos show that cells in the embryonic node are monociliated.

stream of exon 16 . This is expected to generate a protein equivalent to a functional null, as the $\mathrm{N}$-terminally truncated translation product would not contain the motor domain. It is interesting to note that both our Dnabc $5^{d e l 593}$ mutation and the $M d n a b 5^{-/-}$gene trap line share DNA deletion/insertion breakpoints spanning exons 16 and 17, perhaps indicating that this region is of increased instability. The only other mutant mouse line with the full spectrum of defect phenotypes seen in PCD is the Hfh4-knockout mouse, which exhibits situs anomalies, hydrocephalus, and respiratory defects (24). However, so far $\mathrm{HFH}$-4 mutations have not been found in PCD patients (25).

A high incidence of heterotaxy in Dnabc $5^{\text {del593 }}$ mutants. We found a surprisingly high incidence of heterotaxy in homozygous Dnabc5 ${ }^{\text {del593 }}$ mutants. Systematic analysis of 18 litters of embryos showed that $40 \%$ of Dnabc5 $5^{\text {del } 593 / d e l 593}$ mutants exhibited heterotaxy, $35 \%$ situs inversus totalis, and only $25 \%$ situs solitus. Some heterotaxy was also seen in the $M d n a b 5^{-/-}$mutants, but the frequency was not reported (21). In comparison, we note that PCD patients are often said to have $50 \%$ incidence of situs solitus versus situs inversus totalis, although a recent clinical study showed that at least $6.3 \%$ of PCD patients have heterotaxy and congenital heart defects (16). As PCD patients are not routinely examined for heterotaxy or congenital heart defects, the incidence is likely underreported. Based on our findings, we suggest that patients with mutations in DNAH5 should be screened for heterotaxy and congenital heart defect, as complex structural heart defects are a major cause of morbidity and mortality among patients with laterality defects. Conversely, patients with heterotaxy and congenital heart defects should be evaluated for PCD, especially those being considered for cardiac surgery. A diagnosis of PCD would significantly alter the clinical management of surgical patients. We note that some patients with heterotaxy become ventilator dependent after undergoing successful surgical repair of structural heart defects (26). One recent study reported that neonatal respiratory distress can be associated with PCD (27).

Congenital heart defects and heterotaxy. Complex structural heart defects are commonly reported in conjunction with heterotaxy (28). The phenotypes exhibited by the heterotaxic Dnabc5 $5^{\text {del593 }}$ animals recapitulated many of those seen clinically in heterotaxy syndromes, such as pulmonary isomerism, azygos continuation of interrupted IVC, symmetric liver, malrotated gut, and various complex cardiac defects. Although the presentations in the Dnahc5del593 mutants were variable, we rarely observed asplenia and never polysplenia, defects frequently seen in patients with heterotaxy.

As observed in human heterotaxy syndromes, the cardiac anomalies in the $D n a b c 5^{\text {del } 593}$ mutants were highly variable. However, in the Dnahc5del593 mutants, which are genetically homogeneous 
Number of ODAs per cilium

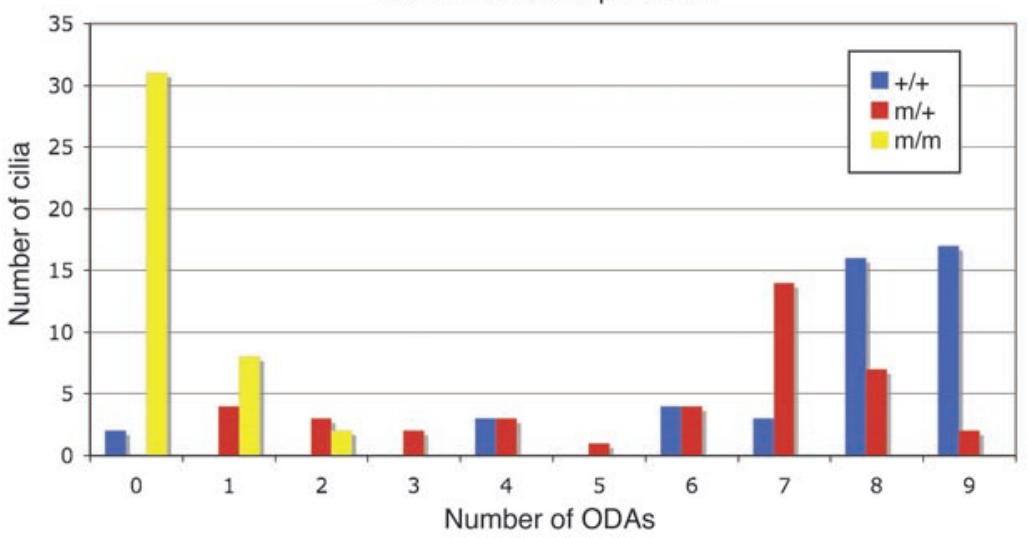

\section{Figure 9}

Graph depicting distribution of ODAs per cilium. ODAs were counted from cross-sectional electron micrographs of cilia from the tracheal epithelia of wild-type, heterozygote, and homozygote mutant animals. The KruskalWallis ANOVA test showed a significant difference in the 3 genotypes, with $P<0.0001$. as compared with the genetic heterogeneity of human patients, the heart anomalies are variations of a defined set of defects, with DORV being one of the most common cardiac malformation. As in humans, a wide spectrum of physiologic consequences may be expected, depending largely on the position of the VSD in relation to the great vessels. One animal had DORV with a VSD committed to the main pulmonary artery (MPA). This would be expected to be deleterious, since most of the oxygenated blood would return to the lungs via the MPA. Other cases of DORV involved VSDs that were committed to the aorta, which would be less severe and similar physiologically to an overriding aorta, such as in TOF.

The nature of individual defects that make up the complex cardiopathies observed in the $D n a b c 5^{\text {del593 }}$ mutants likely reflect the close coupling of left-right determination with various processes in cardiac morphogenesis $(17,29,30)$. Thus, the proper twisting and alignment of the conotruncus over the AV septum is thought to be influenced by the specification of left-right asymmetry. Not surprisingly, more than half of the Dnahc $5^{\text {del593 }}$ mutants with heterotaxy exhibited either DORV or TGA. In DORV, the conotruncus does not migrate sufficiently to the left, resulting in abnormal attachment of the conotruncus on the AV septum such that the right ventricle communicates with both the aorta and the pulmonary artery. TGAs can arise from abnormal twisting of the conotruncus and discordant VA alignment. Aberrant laterality determination also may affect $\mathrm{AV}$ cushion formation and septation, ventricular looping, and atrial situs specification, contributing to the AV canal defects, ASDs, VSDs, L-looped hearts, and atrial isomerism. We also observed a substantial incidence of superior-inferior ventricles among the Dnabc5 $5^{d e l 593}$ mutants exhibiting heterotaxy. The etiology of this is not well understood but has been suggested to arise from excessive twisting of ventricles at the cardiac apex. Together these findings suggest Dnabc5 and cilia function may have roles in patterning of the cardiovascular system beyond the breaking of symmetry and specifying the direction of heart looping.

Role of the cilia in laterality specification. The defects of the Dnabc5 mouse mutants show similarities to those of the well-characterized $i v$ mutant. Both harbor mutations in ciliary dyneins, except that the $i v$ mutation involves the left-right dynein $\operatorname{lrd}(10-13)$. Both Dnabc5 and $i v$ mutations generate randomized asymmetry characterized by situs solitus and situs inversus, and also heterotaxy. Interestingly, a similar percentage (40\%) of $i v / i v$ mice exhibit heterotaxy (10), with heart defects including atrial isomerism, common AV canal, DORV, TOF, TGA, and azygos continuation of interrupted IVC, as well as pulmonary isomerism $(10,11)$ - all phenotypes seen in $D n a b c 5^{\text {del } 593 / d e l 593}$ mutants. In iv/iv mutants, cilia are present at the embryonic node but immotile. Nodal cilia are also present in Dnabc5 ${ }^{\text {del593 }}$ mutants, and given abundant expression of Dnahc5 at the embryonic node (19), we would expect the nodal cilia, like the cilia of the respiratory epithelia, to be dyskinetic or immotile.

The association of ciliary dyskinesia with laterality defects is consistent with the well-described role of the cilia in the specification of laterality $(31,32)$. In Dnabc $5^{\text {del } 593}$ mutant as in $i v / l r d$ mutants, immotile or dyskinetic cilia are associated with randomized laterality, while in inv mutants, reduced leftward nodal flow is associated with situs inversus (33-35). Interestingly, experimental imposition of fast leftward flow across the node rescued most laterality defects in the $i v$ and inv mutants (36-38). In contrast, in Kif $3 A$ - and Kif $3 B$-knockout mice with no nodal cilia, right or left bilateral symmetry is observed with bilateral or absent expression of genes such as pitx2, which is normally expressed only on the left (7-9). These findings suggest that while breaking symmetry requires presence of the nodal cilia, the specification of left-right sidedness also requires ciliary motility.

Table 3

Quantitation of ciliary ultrastructure, orientation and function

$\begin{array}{lc}\text { Genotype } & \text { ODAs/cilium } \\ +/+ & 7.5 \pm 2.1(n=45) \\ \mathrm{m} /+ & 5.7 \pm 2.5(n=40) \\ \mathrm{m} / \mathrm{m} & 0.29 \pm 0.56(n=41), \\ & P<0.0001^{\mathrm{A}}\end{array}$

Ciliary orientation
$6.2^{\circ} \pm 5.1^{\circ}(n=77)$
$7.3^{\circ} \pm 6.5^{\circ}(n=111)$
$12.5^{\circ} \pm 9.7^{\circ}(n=85)$
$P<0.0001^{\mathrm{B}}$

Flow speed $(\mathbf{m m} / \mathbf{s})$
$\begin{aligned} 9.3 & \pm 1.8(n=8) \\ 9.6 & \pm 1.0(n=18) \\ 1.9 & \pm 0.1(n=8), \\ P & =0.0002^{C}\end{aligned}$

\author{
Flow directionality \\ $0.95 \pm 0.03(n=8)$ \\ $0.90 \pm 0.06(n=18)$ \\ $0.37 \pm 0.05(n=8)$, \\ $P<0.0001^{\mathrm{C}}$
}

AKruskal-Wallis ANOVA test shows significant difference between $+/+,+/ \mathrm{m}$, and $\mathrm{m} / \mathrm{m}$ animals. BMann-Whitney $U$ test shows significant difference in ciliary orientation (as measured by mean deviation) between $+/+$ and $\mathrm{m} / \mathrm{m}$ animals. CStastical analysis by ANOVA shows significant differences between $\mathrm{m} / \mathrm{m}$ and $+/+$. 
A recent study suggests this may involve 2 distinct ciliated cell populations (39), with flow generated by motile monociliated cells at the center of the node, which then triggers calcium signaling transduced by mechanosensory function in nonmotile cilia at the node periphery. The resulting asymmetric calcium signaling is thought to activate left-sided expression of nodal, then further activation of downstream transcription factors such as lefty (28) followed by pitx2 (40). We note that the spectrum of phenotypes in the Dnabc5 $5^{\text {del } 593}$ mutants includes left isomerism observed in the lefty-1-knockout mice (41) and right isomerism in mice deficient in Act-RIIB (42), which mediates effects of lefty-2 and nodal. Aberrant expression of lefty-2 and nodal would be predicted to alter pitx 2 expression, with variable situs defects likely a reflection of the differing dosage requirements for pitx2 in different tissues and organs. Indeed, pitx2-knockout mice, like the Dnabc5 $5^{\text {del593 }}$ mutants, exhibit DORV, TGA, AV canal, and septal defects (40). Further studies using the Dnahc $5^{\text {del } 593}$ mutant mouse model should help to elucidate the molecular basis for the complex structural heart defects seen in conjunction with heterotaxy.

\section{Methods}

EFIC histology. All animal studies were conducted under an Animal Study Protocol approved by the National Heart Lung and Blood Institute Animal Care and Use Committee. Mouse fetuses or neonatal stillborn pups obtained from the mating of $D n a h c^{d e l 593 /+}$ mice were fixed in $10 \%$ buffered formalin, and after necropsy, some samples were further processed for paraffin embedding and imaging by EFIC (43). Briefly, using an Orca-ER digital camera, fluorescence images of the block face were captured serially to generate registered $2 \mathrm{D}$ image stacks (43). For 3D volume rendering, the $2 \mathrm{D}$ image stacks were processed using Improvision Volocity software. To refine diagnosis of complex structural heart defects, the $2 \mathrm{D}$ image stacks were viewed in different imaging planes by digital resectioning (43).

Mappings, sequencing, and genotyping analysis. To map the mutation, DNA from mutant embryos in the $\mathrm{B} 6 / \mathrm{C} 3 \mathrm{H}$ hybrid background were genome scanned using $48 \mathrm{~B} 6 / \mathrm{C} 3 \mathrm{H}$ polymorphic microsatellite DNA markers as previously described (18). Further fine mapping was carried out using SNPs within the mapped interval. To analyze Dnabc5 sequences, genomic DNA spanning all 79 exons and the core promoter region of Dnabc5 (mouse genome NCBI Build 36, February 2006; http://www.ncbi.nlm. nih.gov $/$ mapview $/$ stats $/$ BuildStats.cgi? taxid $=10090 \&$ build $=36 \& v e r=1$ ) as well as overlapping sections of Dnabc5 cDNA (GenBank accession number NM_133365) were PCR amplified using Accuprime Pfx polymerase (Invitrogen). Total RNA was prepared (RNEasy kit; QIAGEN) from the heart of mutant embryo exhibiting heterotaxy and PCR amplified after reverse transcription using SuperScript III One-Step HIFI RT-PCR kit (Invitrogen).

Genotyping of the Dnabc5 wild-type and mutant alleles was carried out using Accuprime Pfx polymerase (Invitrogen) with 3 primers targeting the upstream boundary of the deletion in the Dnahc5 gene: forward primer common to wild-type and mutant (5'-GCGTGTATTCATGGCATCCG-3'), reverse primer specific for wild type (5'-CCTAATTGCTCGGTGGAGTTGG-3'), and another reverse primer targeting the inserted sequence $\left(5^{\prime}\right.$ CTGTGCTGGGAAGAAGTTGTGC-3'). PCR reactions were carried out for 35 cycles at an annealing temperature of $55^{\circ} \mathrm{C}$. The wild-type and mutant Dnabc5 genes yielded amplicons of $478 \mathrm{bp}$ and $643 \mathrm{bp}$, respectively.

EM of tracheal epithelial cilia. Trachea were dissected and fixed for 2 hours in $2.5 \%$ glutaraldehyde in $0.12 \mathrm{M}$ sodium cacodylate buffer at $\mathrm{pH} 7.4$ with $2 \mathrm{mM} \mathrm{MgSO}_{4}$ (23), postfixed with $1 \% \mathrm{OsO}_{4}$, followed by en bloc staining with $1 \%$ uranyl acetate and dehydrated to $100 \%$ ethanol containing $1 \%$ tannic acid (23). Thin sections were post-stained with uranyl acetate and lead citrate, then examined with a JEM 1200EX electron microscope. Images were recorded digitally with an ORCA High Resolution Camera (Advanced Microscopy Techniques Corp.). For SEM, trachea were fixed in $2.5 \%$ glutaraldehyde, postfixed in $1 \% \mathrm{OsO}_{4}$, then dehydrated in ethanol. The dehydrated tissue was critical point dried, mounted on aluminum stubs with colloidal silver adhesive, coated with $20 \mathrm{~nm}$ of gold with an EMS 575X sputter coater, and examined with a Hitachi S3400-N1 SEM.

Quantitation of $O D A$ and ciliary orientation. For quantitation of dynein arms, we used transverse sections through the cilia in which the 9 circumferential microtubule doublets could be clearly seen. ODAs were counted only if they projected clearly and continuously from the microtubule doublet in the same plane of focus. Orientation of the cilia was determined using a previously described technique (44) in which a line was drawn through the 2 central microtubules of each cilium, and the angle subtended with the reference line was measured. The mean of the ciliary angles for each field was calculated and subtracted from each measured angle, resulting in a series of differences from the mean ciliary angle with values between -90 and 90 . The mean of these differences is theoretically 0 , and the standard deviation provides a measure of the variation in ciliary beat axis and ciliary deviation.

Quantitation of ciliary motility by videomicroscopy. Trachea were flushed to remove mucus, cut open along one side, and placed lumen side up under a $63 \times$ water immersion lens in L-15 medium with $10 \%$ FBS at room temperature. Fifty microliters of $0.05-\mu \mathrm{m}$ microspheres (catalog 17149; Polysciences) was added, and video images were captured under epifluorescence (excitation 425/60 nm; emission $480 \mathrm{~nm}$ ) using Hamamatsu CCD camera C9100-12 at 15 frames/second. Improvision Volocity 3.5.1 software was used to determine particle speed and directionality. Directionality was defined as net displacement divided by the total distance traveled, with a bead moving in a straight line having directionality of 1 .

Statistics. For the quantitation of ODAs per cilium, 3 populations were compared using the Kruskal-Wallis ANOVA test, showing a variation among the populations with a $P$ value less than 0.0001 . For ciliary orientation the populations were compared using the Mann-Whitney test. This yielded a significant difference between $+/+$ and $\mathrm{m} / \mathrm{m}(P<0.0001)$, but not $+/+$ and $\mathrm{m} /+(P=0.146)$. Flow speed and directionality was found to be significantly altered $(P=0.002)$ when analyzed using 1 -way ANOVA. The Bonferroni test was used to compare individual data points and revealed significant differences between $+/+$ versus $\mathrm{m} / \mathrm{m}(P<0.01)$ and $+/-$ versus $\mathrm{m} / \mathrm{m}(P<0.001)$, while $+/+$ versus $+/-$ was not significantly different.

\section{Acknowledgments}

We are grateful to Amie Batson for technical assistance with electron microscopy. This work was supported by NHLBI grant ZO1HL005701. S.Y. Tan was supported by a Sarnoff Cardiovascular Foundation fellowship. H. Omran was supported by grants from the Deutsche Forschungsgemeinschaft (DFG Om 6/3 and SFB592).

Received for publication July 16, 2007, and accepted in revised form September 26, 2007.

Address correspondence to: Cecilia W. Lo, 10 Center Drive MSC 1583, National Institutes of Health/NHLBI, Bethesda, Maryland 20892-1583, USA. Phone: (301) 451-8041; Fax: (301) 480-4581; E-mail: loc@nhlbi.nih.gov.
1. Afzelius, B.A. 2004. Cilia-related diseases. J. Pathol. 204:470-477.

2. Zariwala, M.A., Knowles, M.R., and Omran, H. 2007. Genetic defects in ciliary structure and func- tion. Annu. Rev. Physiol. 69:423-450.

3. Hornef, N., et al. 2006. DNAH5 mutations are a common cause of primary ciliary dyskinesia with outer dynein arm defects. Am. J. Respir. Crit. Care
Med. 174:120-126.

4. Greenstone, M.A., Jones, R.W., Dewar, A., Neville, B.G., and Cole, P.J. 1984. Hydrocephalus and primary ciliary dyskinesia. Arch. Dis. Child. 59:481-482. 
5. Afzelius, B.A. 1976. A human syndrome caused by immotile cilia. Science. 193:317-319.

6. Sulik, K., et al. 1994. Morphogenesis of the murine node and notochordal plate. Dev. Dyn. 201:260-278.

7. Marszalek, J.R., Ruiz-Lozano, P., Roberts, E., Chien, K.R., and Goldstein, L.S. 1999. Situs inversus and embryonic ciliary morphogenesis defects in mouse mutants lacking the KIF3A subunit of kinesin-II. Proc. Natl. Acad. Sci. U. S. A. 96:5043-5048.

8. Nonaka, S., et al. 1998. Randomization of left-right asymmetry due to loss of nodal cilia generating leftward flow of extraembryonic fluid in mice lacking KIF3B motor protein. Cell. 95:829-837.

9. Takeda, S., et al. 1999. Left-right asymmetry and kinesin superfamily protein KIF3A: new insights in determination of laterality and mesoderm induction by kif3A-/- mice analysis. J. Cell Biol. 145:825-836.

10. Icardo, J.M., and Sanchez de Vega, M.J. 1991. Spectrum of heart malformations in mice with situs solitus, situs inversus, and associated visceral heterotaxy. Circulation. 84:2547-2558.

11. Seo, J.W., Brown, N.A., Ho, S.Y., and Anderson, R.H. 1992. Abnormal laterality and congenital cardiac anomalies. Relations of visceral and cardiac morphologies in the iv/iv mouse. Circulation. 86:642-650

12. Supp, D.M., et al. 1999. Targeted deletion of the ATP binding domain of left-right dynein confirms its role in specifying development of left-right asymmetries. Development. 126:5495-5504.

13. Supp, D.M., Witte, D.P., Potter, S.S., and Brueckner, M. 1997. Mutation of an axonemal dynein affects left-right asymmetry in inversus viscerum mice. Nature. 389:963-966.

14. Gershoni-Baruch, R., Gottfried, E., Pery, M., Sahin, A., and Etzioni, A. 1989. Immotile cilia syndrome including polysplenia, situs inversus, and extrahepatic biliary atresia. Am J. Med. Genet. 33:390-393.

15. Belmont, J.W., Mohapatra, B., Towbin, J.A., and Ware, S.M. 2004. Molecular genetics of heterotaxy syndromes. Curr. Opin. Cardiol. 19:216-220.

16. Kennedy, M.P., et al. 2007. Congenital heart disease and other heterotaxic defects in a large cohort of patients with primary ciliary dyskinesia. Circulation. 115:2814-2821.

17. Kathiriya, I.S., and Srivastava, D. 2000. Left-right asymmetry and cardiac looping: implications for cardiac development and congenital heart disease.
Am J. Med. Genet. 97:271-279.

18. Yu, Q., et al. 2004. ENU induced mutations causing congenital cardiovascular anomalies. Development. 131:6211-6223.

19. Olbrich, H., et al. 2002. Mutations in DNAH5 cause primary ciliary dyskinesia and randomization of left-right asymmetry. Nat. Genet. 30:143-144.

20. Ibanez-Tallon, I., et al. 2004. Dysfunction of axonemal dynein heavy chain Mdnah5 inhibits ependymal flow and reveals a novel mechanism for hydrocephalus formation. Hum. Mol. Genet. 13:2133-2141.

21. Ibanez-Tallon, I., Gorokhova, S., and Heintz, N. 2002. Loss of function of axonemal dynein Mdnah5 causes primary ciliary dyskinesia and hydrocephalus. Hum. Mol. Genet. 11:715-721.

22. Van Praagh, R., Weinberg, P.M., and Van Praagh, S. 1977. Malposition of the heart. In Heart disease in infants, children and adolescents. 2nd edition. A.J. Moss, F.H. Adams, and G.C. Emmanouilides, editors. Baltimore, Maryland, USA. Waverley Press. 394-416.

23. Wilton, L.J., Teichtahl, H., Temple-Smith, P.D., and de Kretser, D.M. 1985. Structural heterogeneity of the axonemes of respiratory cilia and sperm flagella in normal men. J. Clin. Invest. 75:825-831.

24. Brody, S.L., Yan, X.H., Wuerffel, M.K., Song, S.K., and Shapiro, S.D. 2000. Ciliogenesis and left-right axis defects in forkhead factor HFH-4-null mice. Am. J. Respir. Cell. Mol. Biol. 23:45-51.

25. Maiti, A.K., et al. 2000. No deleterious mutations in the FOXJ1 (alias HFH-4) gene in patients with primary ciliary dyskinesia (PCD). Cytogenet. Cell Genet. 90:119-122.

26. Jonas, R.A. 2004. Weaning from mechanical ventilation. In Comprehensive surgical management of congenital heart disease. Oxford University Press. New York, New York, USA. 91-92.

27. Ferkol, T., and Leigh, M. 2006. Primary ciliary dyskinesia and newborn respiratory distress. Semin. Perinatol. 30:335-340.

28. Peeters, H., and Devriendt, K. 2006. Human laterality disorders. Eur. J. Med. Genet. 49:349-362.

29. Harvey, R.P. 2002. Patterning the vertebrate heart Nat. Rev. Genet. 3:544-556.

30. Ramsdell, A.F. 2005. Left-right asymmetry and congenital cardiac defects: getting to the heart of the matter in vertebrate left-right axis determination. Dev. Biol. 288:1-20.
31. Hirokawa, N., Tanaka, Y., Okada, Y., and Takeda, S. 2006. Nodal flow and the generation of left-right asymmetry. Cell. 125:33-45.

32. Levin, M. 2005. Left-right asymmetry in embryonic development: a comprehensive review. Mech. Dev. 122:3-25.

33. McQuinn, T.C., Miga, D.E., Mjaatvedt, C.H., Phelps, A.L., and Wessels, A. 2001. Cardiopulmonary malformations in the inv/inv mouse. Anat. Rec. 263:62-71.

34. Morgan, D., et al. 1998. Inversin, a novel gene in the vertebrate left-right axis pathway, is partially deleted in the inv mouse. Nat. Genet. 20:149-156.

35. Morishima, M., et al. 1998. Situs variation and cardiovascular anomalies in the transgenic mouse insertional mutation, inv. Teratology. 57:302-309.

36. Nonaka, S., Shiratori, H., Saijoh, Y., and Hamada, H. 2002. Determination of left-right patterning of the mouse embryo by artificial nodal flow. Nature. 418:96-99.

37. Watanabe, D., et al. 2003. The left-right determinant Inversin is a component of node monocilia and other 9+0 cilia. Development. 130:1725-1734.

38. Tabin, C.J., and Vogan, K.J. 2003. A two-cilia model for vertebrate left-right axis specification. Genes Dev. 17:1-6.

39. McGrath, J., Somlo, S., Makova, S., Tian, X., and Brueckner, M. 2003. Two populations of node monocilia initiate left-right asymmetry in the mouse. Cell. 114:61-73.

40. Franco, D., and Campione, M. 2003. The role of Pitx2 during cardiac development. Linking leftright signaling and congenital heart diseases. Trends Cardiovasc. Med. 13:157-163.

41. Meno, C., et al. 1998. lefty-1 is required for leftright determination as a regulator of lefty- 2 and nodal. Cell. 94:287-297.

42. Oh, S.P., and Li, E. 1997. The signaling pathway mediated by the type IIB activin receptor controls axial patterning and lateral asymmetry in the mouse. Genes Dev. 11:1812-1826.

43. Rosenthal, J., et al. 2004. Rapid high resolution three dimensional reconstruction of embryos with episcopic fluorescence image capture. Birth Defects Res. C Embryo Today. 72:213-223.

44. De Iongh, R., and Rutland, J. 1989. Orientation of respiratory tract cilia in patients with primary ciliary dyskinesia, bronchiectasis, and in normal subjects. J. Clin. Pathol. 42:613-619. 